\title{
Patch Priority based Region Filling
}

\author{
Darakhshan R. Khan \\ Student, M.E. (Computer Engineering) \\ Thadomal Shahani Engineering College \\ Mumbai, India
}

\author{
Archana B. Patankar, PhD \\ Associate Professor, Computer Department \\ Thadomal Shahani Engineering College \\ Mumbai, India
}

\begin{abstract}
Region filling is the way to fill in the missing region based on the information available from remaining part of the image. The occluded area must be rejuvenated in a visibly persuasive style is the main goal.

This paper proposes a methodology for automating patch priority based region filling process. First, patch sizes are decided based on smallest texture of the image. Region filling algorithms fills in the missing region to create numerous region filled image. Finally, single region filled image is created by amalgamating images.

This arrangement will have an edge over the other region filling algorithms such as patch size will be based on the input image which barbarizes the process of region filling and to deal with sensitivity in region filling, algorithm different parameter settings are used.
\end{abstract}

\section{General Terms}

Image Processing, Image, Region Filling Algorithm, Object Removal.

\section{Keywords}

Object Removal; Region Filling; Patch Priority; Texture Synthesis, Sparsity Based Priority; Inpainitng;

\section{INTRODUCTION}

Rejuvenating the damaged regions of an image and restoring them based on the information available in background is known as Region filling. This need to be done in visibly persuasive way. Non-texture region filling is also termed image interpolation [1]. Digital image region filling tries to simulate this process and executes the entire filling process automatically. The algorithm automatically does this in a way that it looks reasonable to the human eye. Details that are hidden/occluded completely by the object to be removed cannot be recovered by any mathematical method. Therefore the objective for this method is not to recover the original image, but to create some image that has a close resemblance with the original image.

Patch priority based region filling attempts to imbrute the clone tool process. Holes in the image is filled by hunting for the most similar patches in the local neighborhood and replicating the pixel values from most similar patch into holes. Blurring effect present in prior techniques can be reduced by filling the region at the patch level as contrary to the pixel level.

This technique is very valuable with lot of applications in various fields. In the field of art and movie theatre, it is used for reconstructing film, to recover the damage caused due to cracks in portraits and small cut or mark on pictures. It is also used for creative effect by removing certain object, removing red eye effect and for removing logos from picture [1]. In
The blocks which are vanished transmission of images can be restored using this technique. During the transmission of images over a network, there may be some parts of an image that are missing. These parts can then be reconstructed using region filling based on patch priority, for example, in a streaming video. Nonessential objects such as microphones, some unwanted animals, person and logos, stamped dates and text etc. in the image can also be removed [1].

To remove unwanted object and fill the region, patch priority based region filling technique will be used. Firstly, user will select an object to be removed and image without an object will be created. This image is fed as the input to the patch priority based region filling algorithm and output is completely filled image. This algorithm will run multiple times with different parameter setting and images obtained will be combined together to yield a single region filled image.

The remaining part of paper is structured as follows: Section 2 , states current research and literature review. Section 3 explains the patch priority based region filling algorithm which explains confidence term, data term and texture synthesis. Section 4, proposes framework which uses the region filling algorithm along with blending techniques to reduce seam, generating multiple images with different parameters and combining them together to reduce blurring artifact in final image. Section 5, discuss the evaluation of proposed framework. Section 6, provides a concluding remark.

\section{BACKGROUND AND LITERATURE REVIEW}

Nowadays, the region filling technology is one of the most emerging and trending topic in computer graphics. And it has many applications such as restoring cultural heritage, producing extraordinary effects in movie and television, removing redundant and undesirable items etc [2]. Generally, working of most of the region filling methods has following steps, in the first step user will physically select target region from the original image that will be restored. The target region will be rejuvenated automatically, by replicating these regions with the pixel values available from the rest of the image. Currently, there are various methods available for region filling that is also known as image inpainting.

And these methods can be organized as follows:-

- Texture synthesis based region filling.

- $\quad$ PDE based region filling.

- Patch priority based region filling.

\subsection{Texture Synthesis Based Region Filling}

These algorithms fill in the occluded area or target region using analogous proximity of the damaged pixels. In this new 
image pixel synthesis begins from initial seed and then algorithm struggles to maintain the local structure of the image. Most of the prior techniques employ these methods to fill in the occluded area by sampling and copying pixel from closest proximity [2].

The main objective of texture synthesis is to develop texture patterns, which is identical to a given sample pattern. The generated pattern must hold the statistical properties of the parent texture i.e. original image. Due to which there is no tiled rearrangement of the parent texture in the final image [2].

\subsection{PDE Based Region Filling}

Bertalmio et.al has first suggested Partial Differential Equation (PDE) based algorithm for region filling. This algorithm works in a recursive fashion. The vital aim of these algorithms is to propagate geometric and photometric information that is present at the boundary of the target region into the region itself [3]. This is achieved by using isophote lines that is information is proliferated in the direction where change is minimum. When the target region is small, this algorithm produces very effective results. But the quality of result degrades and even it takes longer time to produce output when the target region is large. This method is much useful for noise removal application and for smaller regions. But the main limitation of this method is that it can neither connect separate or disintegrated edges nor can develop good texture patterns [3].

\subsection{Patch Priority Based Region Filling}

Patch priority based region filling is also known as exemplar based approach, an important class of inpainting i.e. region filling algorithms. And they are one of the most efficient region filling algorithms. Generally this category of algorithm has two main steps:

- Patch priority assignment

- $\quad$ Selecting best similar patch for synthesis

The exemplar based approach identifies the identical matching patches from the remaining part of the image, with the help of certain metrics similarity is measured between the patches from known and unknown region and matching patch is copied into the target region. This method works very effectively for restoring larger target regions. Generally, an patch priority based region filling algorithm includes the following four basic steps:

1. Identify and isolate the target region, in which the occluded regions are separated and expressed with relevant data structure.

2. Computing patch priorities, in this an already defined priority function is used to calculate the filling order i.e. priorities of unfilled patches at the start of each filling iteration.

3. Searching best match and propagating, in which the best matching candidate is searched from the source region to replicate and paste the patch in the missing region.

4. Updating target region information, in which the fill front of the target region and the necessary information for calculating patch priorities is updated [2].

Numerous algorithms are developed for the patch priority based region filling such as Criminisi [4] developed an efficient and simple approach that combines the advantages of two approaches. Firstly, Both texture and structure information is propagated with the process of exemplar-based texture synthesis. The filling order highly defines the gain of structure propagation. And then a best-first algorithm in which the confidence of the synthesized patches is replicated in a similar fashion as to the propagation of information in region filling.

The priority function for different algorithm presented in [4] was generalizes by Cheng [5] to give much robust and concrete performance. Wong [6] developed a weighted similarity function. This function makes use of various source patches to rejuvenate the target patch instead of using only a single source patch.

Lignima [7] has developed two-step process. First, it immensely reduces the computational complexity of unbounded searching using gradient-based. Second, the veracity of the best matching patch is increased by using a distance dependent criterion in patch matching process. Fang [8] had use a multi-resolution training method and a patch priority based texture synthesis method to developed a rapid image Inpainting system.

$\mathrm{Xu}$ [9] proposed two unique concepts for calculating the priority of the patch and for representation of patch using Sparsity at the patch level.

In comparison with other approaches, patch priority based region filling approach has acquired splendid output in rejuvenating missing area.

\section{PATCH PRIORITY REGION FILLING ALGORITHM}

The notations used in this paper are same as the one use in [4], which deals with region filling. I denotes the input, i.e. original image. $\Omega$ denotes occluded area or region to be filled $\Phi$ represents the source region, i.e. remaining part of the image which is used to fill missing area. Basically, $\Phi=\mathrm{I}-\Omega$. $\delta \Omega$ represents boundary of the missing region or the target region.

The paper has proposed a system which uses patch priority based region filling which has two basic steps:

- The filling order computation or patch priority

- The texture synthesis

\subsection{Patch Priority}

The filling order computation defines a measure of priority for each patch in order to distinguish the structures from the textures. Basically, the presence of structure is represented by high priority. Given a patch $\Psi \mathrm{p}$ which is centered on pixel $\mathrm{p}$, its priority $\mathrm{P}(\mathrm{p})$ is given by a data and confidence term [4], that is,

$$
P(p)=C(p) \times D(p) .
$$

where $\mathrm{C}(\mathrm{p})$ is the confidence term and $\mathrm{D}(\mathrm{p})$ is the data term.

The confidence term, can be thought as a measure which determines the amount of useful and concrete information surrounding the pixel $\mathrm{p}$, which is define as,

$$
\mathbf{C}(\mathbf{p})=\frac{\sum_{q \in \Psi p \cap \Omega} \mathrm{C}(\mathrm{q})}{|\Psi p|}
$$

Where $|\Psi \mathrm{p}|$ is the area of $\Psi \mathrm{p}$. While initializing, the function $\mathrm{C}(\mathrm{p})$ is set to $\mathrm{C}(\mathrm{p})=0 \quad \forall \mathrm{p} \in \Omega$ and $\mathrm{C}(\mathrm{p})=1 \quad \forall \mathrm{p} \in \mathrm{I}-$ $\Omega$. The patches from the target region which have many of 
their pixels already replete to be fill first is the main aim, with extra importance given to pixels that were filled early on (or which were never the member of the occluded area) [4].

For data term, the Sparsity-based priority will be used. In a search window, a template matching will be performed between the current patch and neighboring patches that belong to the source region of the image. By using a non-local means approach, a similarity weight $\mathrm{w}_{\text {pxpj }}$ (i.e. proportional to the similarity between the two patches centered on $p_{x}$ and $p_{j}$ ) will be computed for each pair of patches [9]. The Sparsity term is defined as:

$$
\mathbf{D}(\mathbf{p x})=\left\|\mathbf{w}_{\mathbf{p x}}\right\|_{2} \times \sqrt{\frac{|\mathrm{Ns}(\mathrm{px})|}{|\mathrm{N}(\mathrm{px})|}}
$$

where Ns and $\mathrm{N}$ represent the number of valid patches (having all its pixels known) and the total number of candidates in the search window. Larger sparseness means this $\left\|\mathrm{w}_{\mathrm{px}}\right\|_{2}$ value is high whereas a small value represents that the current input patch is highly calculable by many other patches in source region [9].

\subsection{Texture Synthesis}

The patch with the highest priority will be filled first. Unknown part of the patch under observation $\psi^{\mathrm{uk}}{ }_{\mathrm{p}}$ will be filled using a similarity measure, for this the most similar patch located in a local neighbourhood $W$ centered on the current patch will be sought. The chosen patch $\psi^{*}$ p maximizes the similarity between the known pixel values of the current patch to be filled in $\psi_{p x}^{\mathrm{k}}$ and co-located pixel values of patches belonging to $W$ :

$$
\begin{gathered}
\psi_{\mathrm{p}}^{*}=\arg \min _{\psi \mathrm{q} \in \mathrm{W}}(\psi \mathrm{p}, \psi \mathrm{q}) \\
\text { s.t } \operatorname{Coh}\left(\psi_{\mathrm{p}}^{\mathrm{uk}}\right)<\lambda_{\text {coh }}
\end{gathered}
$$

where $\mathrm{d}($.$) is the weighted Bhattacharya [8]. \operatorname{Coh}($.$) is the$ coherence measure initially proposed by Wexler et al.

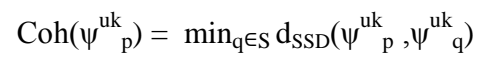

where $d_{S S D}$ is the sum of square differences. The degree of similarity between the synthesized patch $\psi^{\mathrm{uk}}$ and original patches is given by the coherence measure Coh. Due to the constraint in equation (4) the texture which is very different from the root texture will not be copied. If none of the candidates fulfill the constraint (4), the filling process is stopped and the priority of the current patch is decreased. The process restarts by seeking the patch having the highest priority.

\subsection{Recalculating Confidence Values}

After the patch $\psi_{\mathrm{p}}$, has been permeated with new pixel values, the confidence $C$ ( $p$ ) will be updated in the area delimited by $\psi_{\mathrm{p}}$, as follows [4]:

$$
\mathrm{C}(\mathrm{q})=\mathrm{C}\left(\mathrm{p}^{\prime}\right) \quad \forall \mathrm{q} \in \psi_{\mathrm{p}}, \cap \Omega
$$

This simple update rule will allow us to gauge the proportionate confidence of patches on the boundary of target region, without image-specific parameters. As filling algorithm executes, there is a decline in confidence value which represents that we are less sure of the color values of pixels near the center of the target region.

\section{PROPOSED SYSTEM}

The proposed framework consist of five phases : (1) Texture detection (2) Mask creation (3) Patch priority based region filling algorithm (4) Blending methods (5) Combining region filled images.

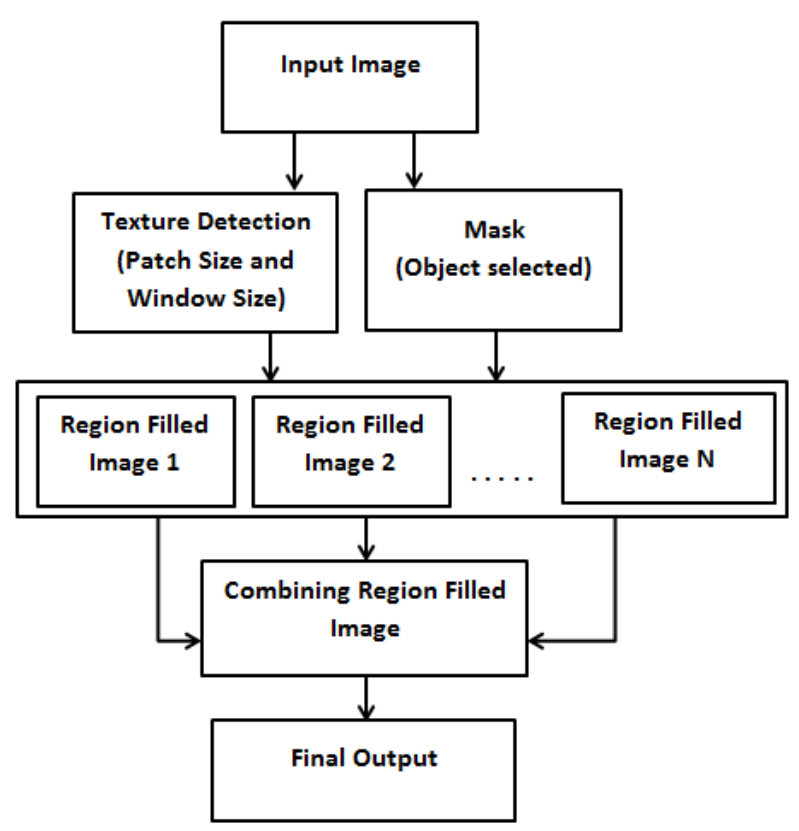

Fig. 1 Proposed framework for region filling

\subsection{Texture Detection}

Practically, the patch size to be used in region filling algorithm must be marginally bigger than the largest perceptible texture element in the source image [4 ]. Image will be fed to the texture detection module to determine the smallest texture, which will eventually decide various parameters. To identify the texture of an image we will identify all the key points in an image since key points denotes specific features or change in texture for an image. The smallest distance between two key points will be treated as the diagonal length of the patch and the most frequent distance will be treated as the window size.

\subsection{Mask Creation}

Object is the region that is to be removed and then filled with the help of information available in the rest of the image. This object could be a complete object that is to eliminated or damage part of the image that is to be repaired. User will manually select object to be removes from the image.

\subsection{Region Filling Algorithm}

Region filling algorithm is explained in section 3, can be summarized into following steps as shown in figure 2 . 


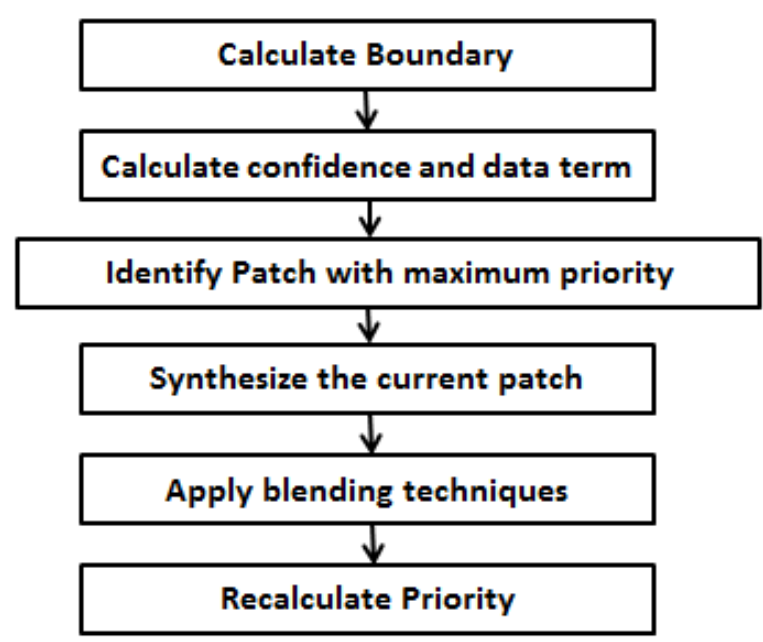

Fig. 2 Region Filling Algorithm

The fill front i.e. boundary of the target region is selected. Priority is computed for all the patches in the target region, then identify the patch with the highest priority. Now, synthesize the current patch by identifying the most similar exemplar from the source region. To reduce the seam in output, blending method will be used after synthesis of each patch. We will be using Poisson fusion and $\alpha$-blending techniques.

A Poisson fusion [12] is applied to hide the seams between known and unknown parts. Poisson blending makes use of system of linear equations, such that the variation between the intensity on boundary between source and missing region is as minute as possible. Also Poisson blending tries to minimize the gradient difference across pixels between the background under source and the source region.

An $\boldsymbol{\alpha}$-blending is also applied to combine known parts of the target and the source patch $\left(\psi_{\mathrm{p}}^{\mathrm{k}} \leftarrow \alpha \psi_{\mathrm{p}}^{\mathrm{k}}+(1-\alpha) \psi_{\mathrm{q}}^{\mathrm{k}}\right.$, with $\alpha=0.75$ ) [11]. It gives more strength and reduce seam by locally regulating the results. Lastly, priorities will be recalculating by updating the confidence term as explained in section 3.3. And, the entire algorithm will be recursively called till the entire region is filled.

\subsection{Combining Multiple Region Filled Image}

One pass greedy algorithm is one of the most common problems with region filling algorithm. To overcome this problem, we will be running region filling algorithm multiple times with different parameter setting such as patch size and filling order. To obtain final single region filled image, average or median operator can be used [10]. Following equations can be used to obtain pixel values:

$$
\mathrm{I}\left(\mathrm{p}_{\mathrm{x}}\right)=\frac{1}{\mathrm{n}} \sum_{\mathrm{i}=1}^{\mathrm{n}} \mathrm{I}^{(\mathrm{i})}\left(\mathrm{p}_{\mathrm{x}}\right)
$$

$$
\mathrm{I}\left(\mathrm{p}_{\mathrm{x}}\right)=\mathbf{M} \mathbf{E} \mathbf{D}_{\mathrm{i}=\mathbf{1}} \text { to } \mathbf{n} \mathrm{I}^{(\mathrm{i})}\left(\mathrm{p}_{\mathrm{x}}\right)
$$

Combining images will definitely reduce the blur effect in the output image. Output will be completely filled image with less blur and seam.

\section{RESULTS}

The results of the few modules that have been implemented are shown in figure 3 , where user will select an object to be removed from the original image and mask will be created.

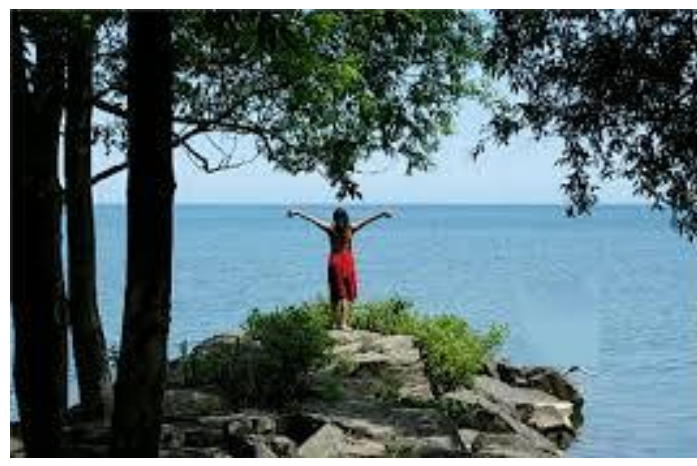

Fig 3 (a). Original Image

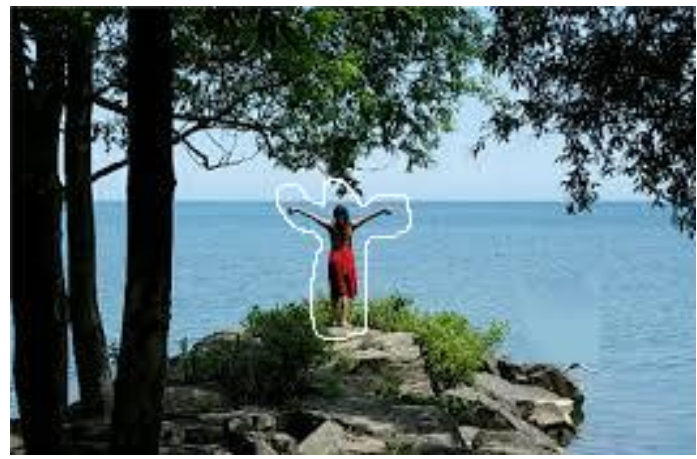

Fig 3 (b). Object Selection

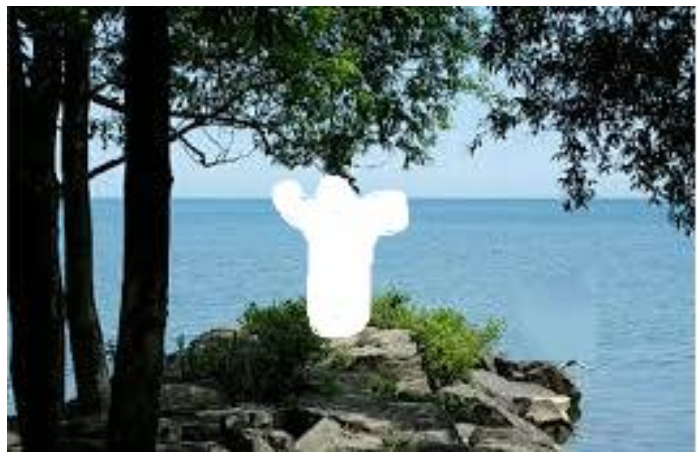

Fig. 3 (c). Mask Creation 
Table 1. Configurations for different region filling images

\begin{tabular}{|c|c|}
\hline Settings & Parameters \\
\hline 1 & $\begin{array}{l}\text { Patch's Size } \mathrm{n} \times \mathrm{n} \\
\text { Search Window } \mathrm{m} \times \mathrm{m} \text { ( where } \mathrm{m} \text { and } \mathrm{n} \text { is } \\
\text { determined by texture detection module). } \\
\text { Sparsity based data term. }\end{array}$ \\
\hline 2 & $\begin{array}{l}\text { Patch's Size } \mathrm{n} \times \mathrm{n} \\
\text { Search Window } \mathrm{m} x \\
\text { Sparsity based data term. } \\
\text { Rotation by } 180 \text { degrees. }\end{array}$ \\
\hline 3 & $\begin{array}{l}\text { Patch's Size } n+2 \times n+2 \\
\text { Search Window } m+5 \times m+5 \\
\text { Sparsity based data term. }\end{array}$ \\
\hline 4 & $\begin{array}{l}\text { Patch's Size } n+2 \times n+2 \\
\text { Search Window } m+5 \times m+5 \\
\text { Sparsity based data term. } \\
\text { Rotation by } 180 \text { degrees. }\end{array}$ \\
\hline 5 & $\begin{array}{l}\text { Patch's Size } n+4 \times n+4 \\
\text { Search Window } m+10 \times m+10 \\
\text { Sparsity based data term. }\end{array}$ \\
\hline 6 & $\begin{array}{l}\text { Patch's Size } n+4 \times n+4 \\
\text { Search Window } m+10 \times m+10 \\
\text { Sparsity based data term. } \\
\text { Rotation by } 180 \text { degrees. }\end{array}$ \\
\hline 7 & $\begin{array}{l}\text { Patch's Size } n+6 \times n+6 \\
\text { Search Window } m+20 \times m+20 \\
\text { Sparsity based data term. }\end{array}$ \\
\hline 8 & $\begin{array}{l}\text { Patch's Size } n+6 \times n+6 \\
\text { Search Window } m+20 \times m+20 \\
\text { Sparsity based data term. } \\
\text { Rotation by } 180 \text { degrees. }\end{array}$ \\
\hline
\end{tabular}

The final module which includes region filling and amalgamation of images to produce single region filled image is yet to be tested.

The most important parameter on which the algorithm will be evaluated is the quality of the region filled image i.e. how plausibly the image has been rejuvenated. Evaluation will be done by performing a psychophysical experiment for subjective i.e. intuitive rating. In this experiment, final region filled image will be conferred to the observers and will request them to inspect the comprehensive quality of the region filled image using the ITU-R five grade quality scale, stamped with the adnoun, Excellent, Good, Fair, Poor, Bad. Based on the obtained visceral data, efficiency of the system can be established [8].

\section{CONCLUSION}

Patch priority based region filling technique is one of the trending and important topics in computer vision and graphics. Region filling algorithm has wide range of application in various domains such as art, theatre, photography, monument preservation, adding special effect etc. The proposed algorithm can fill in the missing region in visibly persuasive fashion.

Uniqueness of this algorithm is it detects patch size based on texture of input image rather than using predefined one. The proposed algorithm calculates priority of patches, highest priority patch is synthesized and patch will be blended in the target region. Multiple images with different parameters setting will be amalgamated to create single region filled image with reduce blurring effect. The proposed method is yet to be evaluated.

The computational time can be further reduced by first creating a low resolution image of original image and working with low resolution image. Lastly, resolution of region filled image can be enhanced to create good quality output.

\section{REFERENCES}

[1] Zhen Xie, Fan Zhang*, Conggui Zhang. 2011. An Adaptive Matching Algorithm for Image Inpainting. International Conference on Electronics, Communications and Control (ICECC).

[2] Komal s Mahajan, Prof. M. B. Vaidya. 2012. Image in Painitng Techniques: A survey. IOSR Journal of Computer Engineering (IOSRJCE).

[3] M. Bertalmio, G. Saprio, V. CAselles, and C. Ballester. 2000. Image Inpainting. In Proceedings of SIGGRAPH, pp. 417-424.

[4] A. Criminisi*, P. P'erez and K. Toyama. 2004. Region Filling and Object Removal by Exemplar-Based Image Inpainting. IEEE Transactions On Image Processing, Vol. 13, No. 9.

[5] SW. Cheng, C. Hsieh, S. Lin, C. Wang and J. Wu. 2005. Robust algorithm for exemplar based image inpainting. In Proceedings of International Conference on Computer Graphics, Imaginig and Visualization, pp.64-69.

[6] A. Wong and J. Orchard. 2008. A non local means approach to exemplar-based inpainting. In Proceedings of the 15 th IEEE International Conference on Image Processin, PP. 2600-2603.

[7] Lingni Ma Luat Do Peter H.N. de With. 2012. Fast and Improved Examplar-Based Inpainting Techniques for Natural Images.

[8] C. Fang and J. J. Lien. 2009. Rapid image completion system using multiresolution patch based directional and nondirectional approaches. IEEE Transactions on Image Processing, Vol. 18, pp. 2769-2779.

[9] Z Xu. And S. Jian. 2010. Image inpainting by patch piopagation using patch sparsity. IEEE Transactions on Image Preocessing, Vol. 19, pp.1153-1165.

[10] Olivier Le Meur, Mounira Ebdelli, and Christine Guillemot. 2013. Hierarchical Super-Resolution-Based Inpainting. IEEE Transactions On Image Processing, Vol. 22.

[11] Olivier Le Meur and Christine Guillemot. 2012. SuperResolution-based Inpainting.

[12] P. Pérez, M. Gangnet, and A. Blake. 2003. Poisson image editing. In Proc.SIGGGRAPH, pp. 313-318.

[13] Alexandra Ioana Oncu, Ferdinand Deger, and Jon YngveHardeberg. 2012. Exaluation of Digital Inpainting Quality in the Context of artwork Restoration. In the journal of Springer-Verlag Berlin Heidelberg. 